\title{
Adiabatic dynamics of quasiperiodic transverse Ising model
}

\author{
Revathy B. S. ${ }^{1}$ and Uma Divakaran ${ }^{1}$ \\ ${ }^{1}$ Department of Physics, Indian Institute of Technology Palakkad, Palakkad, 678557, India
}

(Dated: August 7, 2019)

\begin{abstract}
We study the non-equilibrium dynamics due to slowly taking a quasiperiodic Hamiltonian across its quantum critical point. The special quasiperiodic Hamiltonian that we study here has two different types of critical lines belonging to two different universality classes, one of them being the well known quantum Ising universality class. In this paper, we verify the Kibble Zurek scaling which predicts a power law scaling of the density of defects generated as a function of the rate of variation of the Hamiltonian. The exponent of this power law is related to the equilibrium critical exponents associated with the critical point crossed. We show that the power-law behavior is indeed obeyed when the two types of critical lines are crossed, with the exponents that are correctly predicted by Kibble Zurek scaling.

PACS numbers:
\end{abstract}

\section{INTRODUCTION}

Equilibrium phase diagram of systems undergoing quantum phase transitions (QPT) have been well studied $\underline{\underline{1}} \underline{\underline{3}}$. Recently, studies on non-equilibrium dynamics of a system which is swept linearly with a speed $1 / \tau$ across a quantum critical point has picked up pace mainly due to the following two reasons: (i) the surprising connection between the non-equilibrium exponent and equilibrium exponents $\underline{\underline{4}} \underline{\underline{-6}}$, and (ii) the advancement in the experiments related to optical lattices to simulate quantum Hamiltonians with very high degree of control and accuracy $^{\underline{7}}$. A system at zero temperature which is prepared in its ground state initially, when driven across a quantum critical point by a time dependent Hamiltonian, gets necessarily excited. This is because the relaxation time at the critical point diverges, implying that the system takes infinite time to respond to the external variation. Such a situation results to the system not being able to follow the instantaneous ground state, and hence gets excited. The density of defects $n$ (or excitations) thus generated is related to the speed of linear variation $(1 / \tau)$ and the equilibrium critical exponents by the famous Kibble Zurek (KZ) scaling $n \sim \tau^{-\frac{\nu d}{\nu z+1}}$, where $d$ is the dimensionality of the system, $\nu$ and $z$ are the correlation length and time exponents, respectively, associated with the quantum critical point $\underline{4}^{-6} \underline{6}$. The KZ scaling has been verified in various models and has been accepted as a universal scaling of the density of defects generated as a result of linear variation of a parameter of the Hamiltonian ${ }^{8}$. This scaling can also be generalized to a non-linear variation of a parameter $\underline{9}$.

Quasiperiodic lattices have also gained attention recently as it can have both extended as well as localized states even in one dimensions 10,11 . Till the discovery of quasiperiodic lattices, it was believed that a disordered Hamiltonian can not have extended states in one or two dimensions, but can have only localized states. It is only in three dimensions or higher that a disordered system can have extended states as argued by Anderson $\frac{12}{2}$. On the other hand, it is shown that quasiperiodic lattices, which is intermediate between periodic and disordered systems can still have extended eigenstates 13,14 . The presence of both types of eigenstates lead to an upsurge in the studies related to quasiperiodic system. Quasiperiodic Hamiltonians can also be realized in experiments using lasers of incommensurate lengths $\frac{15}{5}$. Single particle localization in quasiperiodic lattices has already been observed experimentally $\underline{16}$. One such model which has gained a lot of attention is a one-dimensional Aubry Andre model which is essentially an $X X$ spin chain in presence of a quasiperiodic transverse field $\underline{10}$. The beauty of this model is that it undergoes a phase transition from a phase which consists of all extended eigenstates to a phase with all localized eigenstates at a critical value of the transverse field. Moreover, this critical point can be obtained analytically due to its self dual nature when transformed to momentum space $\stackrel{10.11}{ }$. Theoretical studies on non-equilibrium dynamics of Aubry Andre model due to sudden and slow variation of a parameter of the Hamiltonian is studied in Ref. 17 .

Recently, Chandran and Laumann studied a variant of Aubry Andre model, which is Ising model in presence of quasiperiodic transverse field (QPTIM $)^{18}$. The phase diagram of this model is richer and involves possibility of having a mobility edge, i.e., the system can have both extended and localized eigen states at a particular value of Hamiltonian parameters and demonstrate the existence of dynamically stable long range orders which are otherwise not present in equilibrium. They showed the existence of localization protected excited states without disorder. This system also exhibits a new critical line, the dynamical critical properties of which are intermediate between clean Ising critical point and infinite randomness transition point that arises in disordered model. The non-equilibrium dynamics generated due to sudden quenches of QPTIM is already discussed in Ref. 19. We now complete the study of QPTIM by looking at slow variation of a parameter of the Hamiltonian and verify KZ scaling.

This paper is divided as follows: After the basic in- 
troduction to the field of $\mathrm{KZ}$ scaling and quasiperiodic Hamiltonian in Section I, we present a brief description of the Hamiltonian along with the proposed phase diagram in Section II. The non-equilibrium dynamics due to adiabatic evolution of the QPTIM is studied in Section III. We conclude the paper with discussions in Section IV.

\section{THE MODEL AND PHASE DIAGRAM}

The Hamiltonian of QPTIM ${ }^{18}$ is given by

$$
\begin{aligned}
& H=-\frac{1}{2} \sum_{j} J_{i} \sigma_{i}^{x} \sigma_{i+1}^{x}+h \sigma_{i}^{z}, \\
& J_{i}=J+A_{J} \cos (Q(i+1 / 2))
\end{aligned}
$$

with $\sigma_{i}^{\alpha}$ being the Pauli matrices at site $i$, and $\alpha$ corresponding to $x, y$, or $z$. We choose $Q$ to be the golden ratio $Q=2 \pi(\sqrt{5}+1) / 2$, so that the interaction term has a periodicity which is incommensurate with the lattice. The zero temperature phase diagram of this model as obtained by Chandran et.al in $J / h-A_{J} / h$ plane is presented in Fig 1. It consists of three phases, namely, paramagnet (PM), ferromagnet (FM) and quasiperiodically alternating ferromagnet (QPFM). The excited states of the model can be either localized, extended or critical depending upon the values of $J$ and $A_{J}$. The critically delocalized phase consists of states having multifractal scaling behavior. These properties of the eigenstates are obtained analytically wherever symmetry permits and numerically otherwise, the details of which we shall briefly discuss later. The dashed diagonal line in Fig. 1 separates two different PM phases based on their low lying excitations-extended or critical. The thick line originating from $J / h=1$ corresponds to a phase transition belonging to the Ising universality class (marked as $A$ in Fig. 1) with $\nu=1$ and $z=1$. On the other hand, the second phase boundary separating critical PM and localized QPFM belongs to a different universality class (marked B in Fig. 1) with the same correlation length exponent as quantum Ising critical point i.e., $\nu=1$, but with the dynamical exponent $z$ equal to 2 . Therefore, if the adiabatic dynamics involve crossing of the critical line "A", the defect density will follow $\tau^{-0.5}$. On the other hand, if the critical line "B" is crossed, the defect density would give a new exponent with $n \sim \tau^{-0.33}$. In this work, we verify Kibble Zurek scaling while crossing both these different types of critical lines.

For the sake of completeness, we now present a brief discussion on how the phase diagram of this Hamiltonian is obtained in Ref. 18. Owing to the complexity of the Hamiltonian, the form of the eigenstates and corresponding eigen energies is almost impossible to calculate analytically for all parameter values. Therefore, the properties of the phase diagram has been obtained analytically only under certain limits which possess some symmetry, other parts of the phase diagram being obtained numerically. Such special limits include $A_{J}=0$,

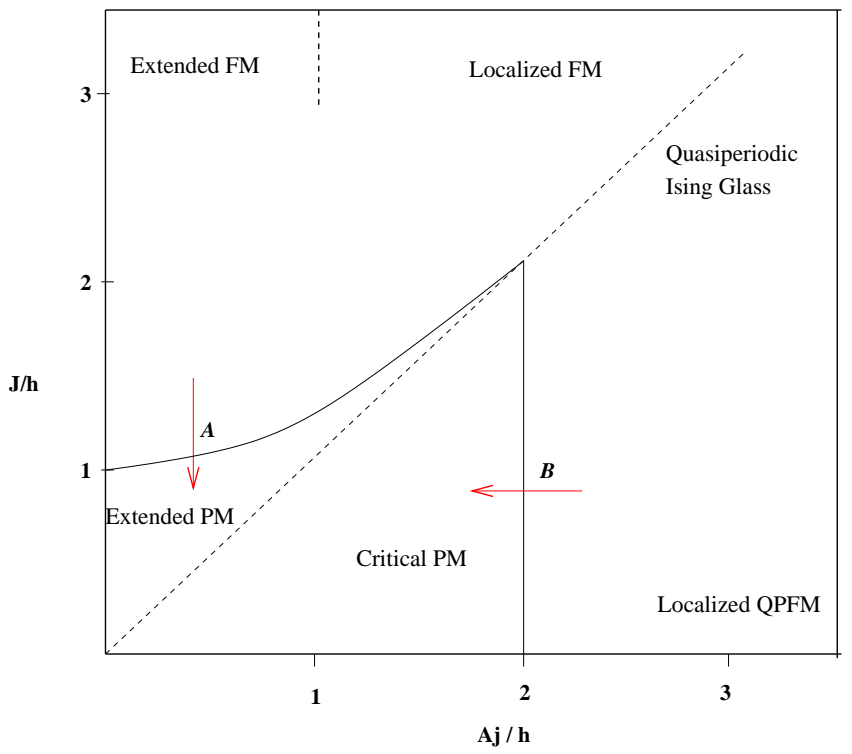

FIG. 1: Phase diagram of QPTIM consisting of FM, PM and QPFM ground states. Depending upon the strength of the quasiperiodic modulation, the low energy excitations can exhibit localized, extended or critically delocalized behavior, also shown in the figure. For more details, see Ref. 18.

$J=0$ and $J \rightarrow \infty$. We consider the limit of $J \rightarrow \infty$ first. It is easy to observe that the ground state of the model in this limit consists of all spins pointing either along $+x$ or $-x$ direction. One can then re-write the Hamiltonian in terms of domain wall dynamics resembling AA model which helps to extend the information about AA model to this model. Such a correspondence allows us to conclude that all the states for $A_{J}<h$ are extended, whereas they are localized for $A_{J}>h$ similar to AA model. This particular limit is shown as the dashed vertical line in Fig. 11 Lets consider the next point $A_{J}=0$ and $J / h=1$ which is the well studied critical point of Transverse Ising model (TIM). It is well known that the transverse Ising Hamiltonian has gapless extended excitations at all energies. As seen in Fig. 11. there is a parabolic phase boundary originating from TIM critical point and extending upto $A_{J} / h=2$. It has been argued using Harris-Luck criterion and also numerically verified that the weak quasiperiodic modulation is irrelevant at small $A_{J}$. Therefore, this parabolic phase boundary belongs to the same universality class as that of $A_{J}=0$, i.e., that of transverse field Ising model. One expects atleast the low lying excitations along this phase boundary to be extended. On the other hand, at $J=0$ and $A_{J} / h=2$, there exist a triality similar to the AA duality found in Aubry Andre model. Using this triality, it can be shown that there is a phase transition from critically delocalized to localized states at all energies at $A_{J} / h=2$. All states are localized for $A_{J} \gg J, h^{18}$. It is to be noted that the localization properties are generally claimed to be $Q$ and energy dependent, which can be cross-checked through numerics. For more details, please 
refer to Ref. 18 .

\section{ADIABATIC DYNAMICS}

Let us now discuss the non-equilibrium dynamics generated as a result of linear time evolution of the transverse field. It is to be noted that the phase diagram discussed till now and given in Ref. 18 is in $J-A_{J}$ plane where the quasiperiodicity is in $A_{J}$ with no quasiperiodicity in $h$. In this paper, for the ease of numerical calculations, we have modified the Hamiltonian bringing the quasiperiodicity in the transverse field, setting $J$ constant and $A_{J}=0$. The Hamiltonian that we numerically simulate has the following form

$$
\begin{aligned}
H & =-\frac{1}{2} \sum_{i} J \sigma_{i}^{x} \sigma_{i+1}^{x}+h_{i} \sigma_{i}^{z}, \\
h_{i} & =h+A_{h} \cos (Q(i+1 / 2))
\end{aligned}
$$

which is exactly like Eq. 1 with quasiperiodicity in $J$ shifted to $h$. It is to be noted that due to the Ising duality in the model, such a change will result into the paramagnetic and ferromagnetic phases getting swapped leaving the dynamical nature of bulk single particle excitations unaltered. Quasiperiodicity in $h$ along with $J$ is also discussed in Ref. 20 by the same authors as in Ref. 18. The phase diagram is then in $h / J-A_{h} / J$ plane with paramagnet replaced by ferromagnet and QPFM replaced by quasiperiodic paramagnet. The critical lines "A" and "B" will still be present separating the corresponding similar phases. We shall continue to call these critical lines as "A" and "B", though they are now in a different plane.

We shall first check the non-equilibrium exponent when the quantum Ising critical line (marked as A in Fig. 10 is crossed where the defect density is expected to decay as $\tau^{-1 / 2}$. To start with, the system is in its ground state at $t=0$. A parameter of the Hamiltonian is varied linearly such that the critical point is crossed during the evolution. Close to the critical point when the relaxation time is larger than the time scale in which the Hamiltonian is varied, the system is no longer able to follow its ground state, and gets excited. Below we describe two different quenching protocols for crossing the above described critical lines. As mentioned before, these protocols are presented in a different plane, i.e., in $h-A_{h}$ plane as opposed to Fig. 11. but the new phase diagram will have similar features due to the Ising duality. To cross the quantum Ising critical line, we divide the evolution into two steps as follows: (i)start deep in the paramagnet phase with $h=5$ and $A_{h}=0.1$ and reduce $h$ to zero linearly as $t / \tau$ which results to crossing of Ising like critical line. (ii)Next, we reduce $A_{h}$ to zero with the same rate. The final Hamiltonian after both the steps is simply Ising Hamiltonian with zero transverse field . Had the evolution been a complete adiabatic evolution, the final state arrived would be all spins parallel to each other along the Ising direction, and any deviation from this would correspond to defects, which in this case are domain walls. It is easy to see that the density of domain walls can be calculated using the following equation ${ }^{21,22}$

$$
n=\frac{1}{L} \sum_{i}^{L-1}\left\langle\psi_{f}\left|\frac{1}{2}\left(1-\sigma_{i}^{x} \sigma_{i+1}^{x}\right)\right| \psi_{f}\right\rangle .
$$

where $\left|\psi_{f}\right\rangle$ is the final evolved state. The visual realization and ability to write a mathematical expression for the defects is the main reason for quenching the system to Ising Hamiltonian. As expected, the numerically obtained density of defects after solving Schrödinger equation follows $n \sim \tau^{-1 / 2}$ behavior characteristic of quantum Ising critical point. This is also shown in Fig. 2. There are two features of this figure. The better agreement with the expected power-law for larger system sizes and the faster decay of defect density for larger $\tau$ values. This can be attributed to the finite size effects. The gap is non-zero for a finite system even at critical point. Therefore, it is always possible to find some $\tau$ value beyond which the evolution is perfectly adiabatic. In summary, we need infinite system to get perfect $\tau^{-1 / 2}$ for all $\tau$ values.

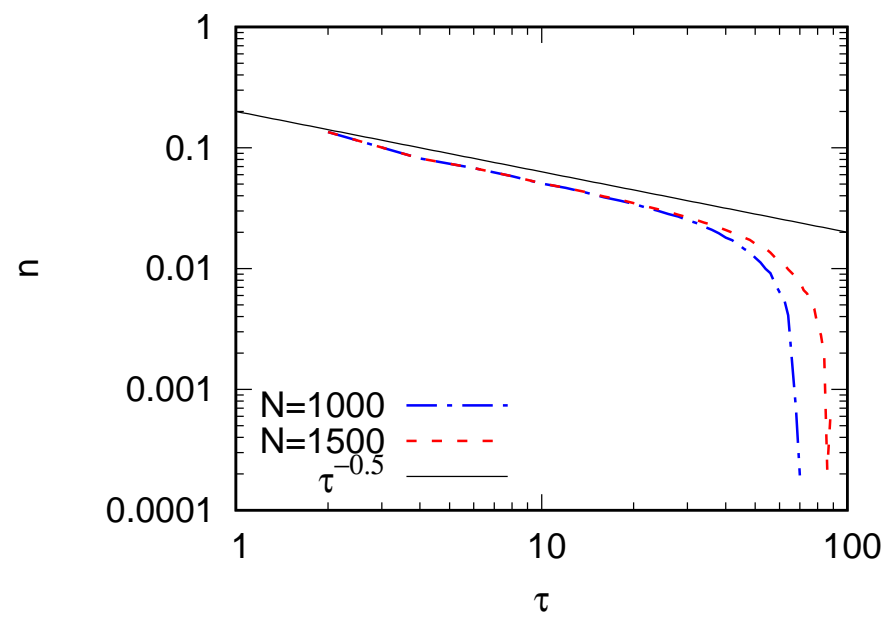

FIG. 2: Variation of defect density with $\tau$ when the critical line "A" is crossed. KZ scaling corresponding to quantum Ising critical exponents predict $n \sim \tau^{-0.5}$ behavior. As seen above, the defect density approaches $\tau^{-0.5}$ for larger system sizes.

Let us now look into the exponent when the "B" critical line is crossed. This is a new type of critical line with a new exponent. For this, the time evolution of the Hamiltonian is divided into two steps: (i) Setting $h$ to 0.1 , we reduce $A_{h}$ as $t / \tau$ from 3 to zero crossing the critical point. (ii) In the next step, we reduce the value of $h$ from 0.1 to zero so that at the final time of the evolution, the Hamiltonian is once again simply Ising Hamiltonian resulting to same expression for $n$ as given in $\mathrm{Eq} 3$. This protocol ensures that only one critical point belonging to 
line "B" is crossed. The critical exponents of the corresponding critical point gives a defect density given by $n \sim \tau^{-1 / 3}$, which is also supported by numerics as shown in Fig. 3, In both the figures, one can clearly see power law behavior, the exponent of which approaches the theoretical value as the system size increases.

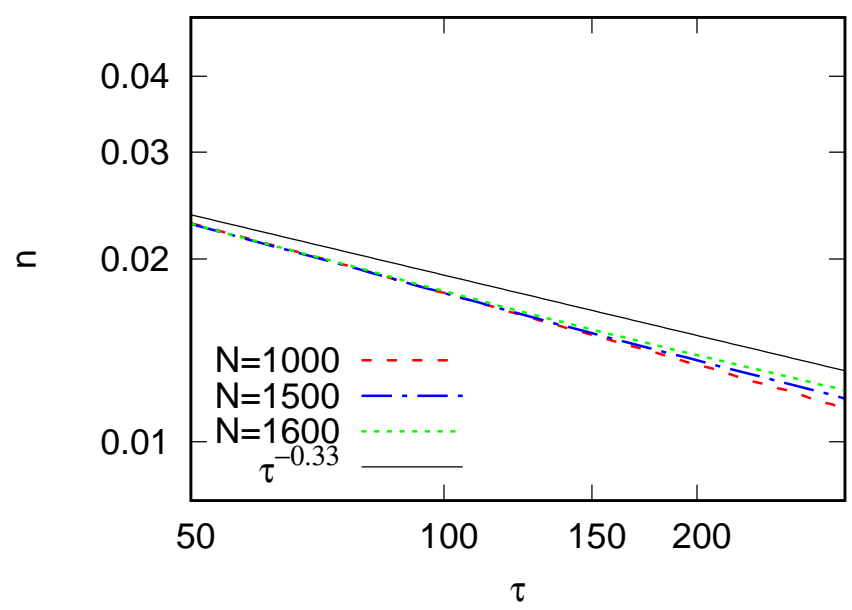

FIG. 3: Variation of defect density with $\tau$ when the critical line "B" is crossed. The defect density $n$ clearly decays with a power law with the exponent close to $1 / 3$. The agreement with the exponent increases as the system size increases.

\section{CONCLUSION}

We have studied the non-equilibrium dynamics after taking the system through two different critical points belonging to two different universality classes. We find that the conventional Kibble Zurek scaling is obeyed in both the cases. This work confirms the equilibrium critical exponents obtained in Ref. 18 for this new model along with the KZ scaling. The numerical calculations clearly show an improvement in the obtained power law scaling as the system size is increased. A thorough study of phase diagram with non-zero $A_{h}$ and $A_{J}$ has been done recently in Ref. 20. It would be interesting to explore this rich phase diagram in connection with non-equilibrium dynamics also.

\section{Acknowledgments}

UD acknowledges DST-INSPIRE Fellowship by Government of India for financial support.
1 S. Sachdev, Quantum Phase Transitions (Cambridge University Press, Cambridge, UK, 1999).

2 B. K. Chakrabarti, A. Dutta and P. Sen, Quantum Ising Phases and transitions in transverse Ising Models, m41 (Springer, heidelberg, 1996).

3 S. Suzuki, J-i Inoue and Bikas K. Chkarabarti, Quantum Ising Phases and Transitions in Transverse Ising Models (Springer, Lecture Notes in Physics, Vol. 862 (2013)).

4 A. Polkovnikov, K. Sengupta, A. Silva, and M. Vengalattore, Rev. Mod. Phys. 83, 863 (2011).

5 J. Dziarmaga, Advances in Physics 59, 1063 (2010).

6 A. Dutta, G. Aeppli, B. K. Chakrabarti, U. Divakaran, T. Rosenbaum and D. Sen, Quantum Phase Transitions in Transverse Field Spin Models: From Statistical Physics to Quantum Information (Cambridge University Press, Cambridge, 2015).

7 I. Bloch, J. Dalibard, and W. Zwerger, Rev. Mod. Phys. 80, 885 (2008).

8 V. Mukherjee, U. Divakaran, A. Dutta, and D. Sen, Phys. Rev. B 76, 174303 (2007); U. Divakaran, V. Mukherjee, A. Dutta, and D. Sen, J. Stat. Mech. P02007 (2009);U. Divakaran, A. Dutta, and D. Sen, Phys. Rev. B 78, 144301 (2008); V. Mukherjee and A. Dutta, EPL 92 , 37004 (2010).

9 S. Mondal, K. Sengupta and D. Sen, Phys. Rev. B 79, 045128 (2009).

10 S. Aubry and G. André, Ann. Israel Phys. Soc. 3133 (1980).
11 P. G. Harper, Proc. Phys. Soc. A 68, 874 (1955).

12 P. W. Anderson, Phys. Rev. 109, 1492 (1958).

13 M. Modugno, New J. Phys. 11033023 (2009);

14 B. Deissler, E. Lucioni, M. Modugno, G. Roati, L. Tanzi, M. Zaccanti, M. Inguscio and G. Modugno, New J. Phys. 13, 023020 (2011).

15 M. Schreiber, S. S. Hodgman, P. Bordia, H. P. Lüschen, M. H. Fischer, R. Vosk, E. Altman, U. Schneider, and I. Bloch, Science 349, 842 (2015); S. S. Kondov, W. R. McGehee, W. Xu and B. DeMarco, Phys. Rev. Lett. 144, 083002 (2015); P. Bordia, H. P. Lüschen, S. S. Hodgman, M. Schreiber, I. Bloch and U. Schneider, Phys. Rev. Lett 116, 140401 (2016).

16 L. Dal Negro, C. J. Oton, Z. Gaburro, L Pavesi, P Johnson, A Lagendijk, R. Righini, M. Colocci and D. S. Wiersma, Phys. Rev. Lett 90, 055501 (2003); G. Roati, C. DErrico, L. Fallani, M. Fattori, C. Fort, M. Zaccanti, G. Modugno, M. Modugno, M. Inguscio, Nature 453, 895 (2008); Y. Lahini, R. Pugatch, F. Pozzi, M. Sorel, R. Morandotti, N. Davidson and Y. Silberberg, Phys. Rev. Lett 103, 013901 (2009); G. Modungo, Rep. Prog. Phys. 73, 102401 (2010).

17 Gergo Roosz, Uma Divakaran, Heiko Rieger and Ferenc Igloi, Phys. Rev. B (2014)

18 A. Chandran and C. R. Laumann, Phys. Rev. X. 7, 031061 (2017).

19 U. Divakaran, Phys. Rev. E (2018)

20 P J. D. Crowley, A. Chandran and C. R. Laumann, Phys. Rev. Lett. 120, 175702 (2018). 
21 J. Dziarmaga, Phys. Rev. Lett. 95, 245701 (2005)

144427 (2007).

22 T. Caneva, R. Fazio, and G. E. Santoro Phys. Rev. B 76, 